\title{
STATUS OF THE VISIBLE FREE-ELECTRON LASER AT THE BROOKHAVEN ACCELERATOR TEST FACILITY*
}

\author{
K. Batchelor, I. Ben-Zvi, R.C. Fernow, A.S. Fisher, A. Friedman \\ J. Gallardo, G. Ingold, H. Kirk, S. Kramer, L. Lin, J.T. Rogers \\ J.F. Sheehan, A. van Steenbergen, M. Woodle, J. Xie, L.H. Yu, R. Zhang \\ Brookhaven National Laboratory, Upton NY 11973 \\ and
}

A. Bhowmik

Rockwell International/Rocketdyne Division, Canoga Park, CA 91303

\begin{abstract}
The $500 \mathrm{~nm}$ Free-Electron Laser (FEL) at the Accelerator Test Facility (ATF) of the Brookhaven National Laboratory is reviewed. We present an overview of the ATF, a highbrightness, $50-\mathrm{MeV}$, electron accelerator and laser complex which is a users' facility for accelerator and beam physics. A number of laser acceleration and FEL experiments are under construction at the ATF. The visible FEL experiment is based on a novel superferric 8.8 $\mathrm{mm}$ period undulator. The electron beam parameters, the undulator, the optical resonator, optical and electron beam diagnostics are discussed. The operational status of the experiment is presented.
\end{abstract}

\section{DISCLAIMER}

\begin{abstract}
This report was prepared as an account of work sponsored by an agency of the United States Government. Neither the United States Government nor any agency thereof, nor any of their employees, makes any warranty, express or implied, or assumes any legal liability or responsibility for the accuracy, completeness, or usefulness of any information, apparatus, product, or process disclosed, or represents that its use would not infringe privately owned rights. Reference herein to any specific commercial product, process, or service by trade name, trademark, manufacturer, or otherwise does not necessarily constitute or imply its endorsement, recommendation, or favoring by the United States Government or any agency thereof. The views and opinions of authors expressed herein do not necessarily state or reflect those of the United States Government or any agency thereof.
\end{abstract}

- This work was done under the auspices of the U.S. Department of Energy under contract number DE-AC02-76-CH0016 


\section{INTRODUCTION}

A Free-Electron Laser (FEL) dedicated to FEL physics research is nearing completion at Brookhaven National Laboratory's Accelerator Test Facility (BNL-ATF). The ATF is a users' facility for accelerator and beam physics. The ATF includes a high-brightness photocathode rf gun, a $50 \mathrm{MeV}$ rf-linac, several high power lasers and associated diagnostics, all available under one roof. The principal research objectives of the FEL experiment are as follows: (i) development of mini-undulators and compact FEL's for the production of visible radiation (ii) advancement of FEL technology to produce ultraviolet radiation [1], [2],[3], and (iii) exploration of the short-wavelength limits for high-brightness electron guns.

At the heart of this device is a powerful and very compact, superconducting miniundulator [4] [5]. The undulator is approximately $60 \mathrm{~cm}$ long and has a period $\lambda_{W}=$ $0.88 \mathrm{~cm}$. In the more commonly used permanent magnet undulators, $\lambda_{W}$ is typically 2.0 - $3.0 \mathrm{~cm}$, or more, and a much longer undulator $(135 \mathrm{~cm}-300 \mathrm{~cm})$ would be required to achieve comparable performance. This compact undulator allows the use of an optical resonator that is also quite compact with a length of $3.7 \mathrm{~m}$. This is short compared to the resonators used at Orsay [6] (18 m), Novosibirsk [7] (18.7 m), and Boeing [8] (60 m). The resonator length in an FEL may be determined by a number of constraints, mechanical as well as other conditions of the experiment. However, a short undulator offers the potential for a compact resonator.

The visible FEL will be used in other ATF experiments such as a Columbia University experiment designed to study spiking in the FEL output pulse and MIT's room temperature, pulsed microundulator experiment.

In this paper we or e a brief description of the ATF and discuss the FEL design including: 3-D simulation res $\ldots . .$. , he undulator, the optical resonator, the precision alignment system and diagnostics. 


\section{THE ACCELERATOR TEST FACILITY}

The ATF [9] is a linac-laser complex for research on the interaction of intense electromagnetic radiation and high-brightness electron beams. Experiments in laser acceleration, Free-Electron Lasers and radiation-electron nonlinear interactions are approved by a users' Committee of the BNL Center for Accelerator Physics.

The electron source is a $1 \frac{1}{2}$ cell, $\pi$-mode, side-coupled rf structure [10] [11] operating at $2.856 \mathrm{GHz}$. The design peak cathode electric field is $100 \mathrm{MV} / \mathrm{m}$ at $6.2 \mathrm{MW}$ input power resulting in a beam energy of $4.6 \mathrm{MeV}$. The gun's photo-cathode is driven by a pulsed, quadrupled Nd:YAG laser. The cathode material is a robust yttrium or copper metal installed on the back plane of the half cell. The design performance calls for 1-nC, 6-ps-long (FWHM), single pulses, or a train of $0.5 \mathrm{nC}$ pulses at a repetition rate of $40.8 \mathrm{MHz}$ (every 70 th rf bucket) in a $2.5-\mu$ s macropulse.

The measured e-beam normalized rms emittance at the exit of the gun [11] is $4 \pi \mathrm{mm}$ mrad. The normalized emittance may grow by a factor of two in the beam transport system, thus we use a more conservative value of $\epsilon \mathrm{v}=8 \pi \mathrm{mm}$ - $\mathrm{mrad}$ in the FEL simulations.

The linac, based on the SLAC design, consists of two s-band sections, each $3 \mathrm{~m}$ long. The beam will be accelerated to $50 \mathrm{MeV}$ by using a single $25 \mathrm{MW}$ klystron (of which up to $12 \mathrm{MW}$ is available to power the rf gun). A future upgrade to $100 \mathrm{MeV}$ is planned through the use of two klystrons. The macropulse repetition rate is 1 to $6 \mathrm{~Hz}$.

The instantaneous energy spread of the electron beam is expected to be better than $\delta \gamma / \gamma$ $=0.3 \%$ rms. Furthermore, a novel adaptive control system [12], under development at the ATF, is expected to produce a similar value for the overall (macropulse) energy stability.

The electron beam transport makes use of a short transport line downstream of the linac, with the possibility of transverse emittance selection by of a series of collimators. Achromatic translation systems bring the beam to any one of three beam lines.

Typical Twiss parameters at the FEL interaction region (neglecting in a first approach the vertical focussing contribution of the undulator), are $\beta_{x}^{*}=\beta_{y}^{*}=0.2, \alpha_{x}=\alpha_{y}=0, \eta=\eta^{\prime}=0$, 
leading to local beam sizes $\sigma_{x}=\sigma_{y}=0.125 \mathrm{~mm}$. With seven selected quadrupoles, (the transport system incorporates 10 quadrupoles between the source location and undulator interaction region), beta function variations in the range of $0.1 \leq \beta_{x, y}^{*} \leq 1 \mathrm{~m}$ with $\alpha_{x}=\alpha_{y}=$ $\eta=\eta^{\prime}=0$ can be achieved.

The vertical focussing of the undulator is taken into account by an approximate expression for the "focussing strength", $K_{y}=1 / 2 \rho^{2}$, where $\rho$ is the bending radius at the maximum field value of the undulator, i.e., $\rho=\lambda_{W} \gamma / 2 \pi K$. The associated period of the betatron oscillation in the extent of the undulator is $\lambda_{\beta}=2 \pi / \sqrt{K_{y}}=2 \sqrt{2} \pi \rho$ with an equivalent local beta function amplitude of $\beta_{W}=\lambda_{\beta} / 2 \pi=\sqrt{2} \rho$, the undulator's 'natural' beta function value.

The deviation of off-axis electrons from the FEL resonance condition is minimized by matching $\beta_{y}^{*}$ to $\beta_{W}$ at the FEL interaction region [13]. With vertical undulator focussing then taken into account and for the case of $\beta_{W}$ ('nat.') $=0.5 \mathrm{~m}$ (i.e. $B_{W}=0.47 \mathrm{~T}, \gamma=100, \rho$ $=0.354 \mathrm{~m}$ ) optical matching solutions were obtained for a range of parameters characterized by $0.2 \leq \beta_{x, y}^{*} \leq 0.8 \mathrm{~m}$ and $\alpha_{x}=\alpha_{y}=\eta=\eta^{\prime}=0$.

With a minor refinement in the optical matching it is also possible to obtain transport isochronism. Typical final path length differences were $\Delta t= \pm 0.03 \mathrm{psec}$ for $\delta p / p= \pm 0.3 \%$. Taking into account the electron microstructure pulse width of $\Delta_{t}=6$ ps (FWH.M), this is clearly negligible.

The beam line includes an array of high-precision electron beam diagnostics in order to characterize and control the beam to high stability. Non-destructive high-sensitivity stripline beam position monitors are used as the primary position diagnostic throughout the line. One stripline monitor is placed at each end of the undulator to maintain alignment of the electron beam after initial alignment using destructive phosphor screen monitors. A stripline monitor will also be used to monitor the mean energy of the beam for use with the adaptive energy control system.

A fast beam profile monitor has been developed which will allow the measurement of the position or energy profile of each micropulse in the pulse train. These monitors will be used 
before and after the FEL undulator to measure the incoming and outgoing electron energy distributions. In addition, the beam line includes two types of profile monitors using CCD cameras to image a phosphor screen. High resolution profile monitors are being used in pairs to measure the electron beam emittance. Lower resolution profile monitors are positioned at many beamline locations to allow us to tune the beamline elements and to provide an additional energy distribution diagnostic. A fast vertical kicker magnet will be used with these to allow resolution of individual micropulses. Each slit and collimator also serve as a profile monitor.

The beamline terminates with a Faraday cup. All signals from the diagnostic devices are digitized and transferred to the control computer via CAMAC standard cards. The video signals undergo intermediate frocessing by a commercial microprocessor-based analyzer.

\section{FEL DESIGN CONSIDERATIONS}

The geometrical emittance at the initial design energy of $50 \mathrm{MeV}$ sets the FEL's short wavelength limit, $\lambda \leq 2 \pi \epsilon$. Taking a conservative estimate of the emittance at the FEL interaction region $\epsilon=\sigma_{y} \sigma_{y}^{\prime} \sim 0.08 \pi \mathrm{mm}-\mathrm{mrad}$, the shortest laser wavelength one can expect from the ATF-FEL (without compromising its performance) is around $0.5 \mu \mathrm{m}$.

For a given beam energy $\gamma$, the laser wavelength determines the undulator period, $\lambda_{W} \sim$ $2 \gamma^{2} \lambda$, which yields $\lambda_{W} \sim 0.8 \mathrm{~cm}$ (for $\gamma=100$ ).

The linac energy spread determines the maximum useful length of an undulator, $L_{W}^{\max }=$ $N_{W} \lambda_{W}$, where the number of undulator periods is $N_{W} \sim \gamma /(2 \delta \gamma)$, and $\delta \gamma / \gamma$ is the fractional beam energy spread. Assuming the energy spread to be maintained at $\delta \gamma / \gamma \sim 5 \times 10^{-3}$, we get $N_{W} \sim 100$, so that $\left(L_{W}\right) \max \sim 80 \mathrm{~cm}$.

The desired small signal gain, $G$, then determines the peak undulator field $B_{W}$ for a given beam current density (or electron number density). The maximum value of the small signal gain for a plane-polarized, constant-period undulator driven by a monoenergetic beam is given (in cgs units) by [14] $G_{\max }=0.135 e^{4} B_{W}^{2} \lambda_{W} \rho_{0}\left(L_{W} / \gamma m c^{2}\right)^{4}\left[J_{0}(x)-J_{1}(x)\right]^{2}$ where e 
and $\mathrm{m}$ are the electron charge and mass, respectively, $\rho_{0}$ is the electron number density, $x=\frac{1}{2} \frac{K^{2} / 2}{1+K^{2} / 2},\left(K=0.934 B_{W}(T) \lambda_{W}(\mathrm{~cm})\right.$ is the undulator strength $)$ and the difference in Bessel functions $\left[J_{0}(x)-J_{1}(x)\right]$ is of the order of unity. Assuming $B_{W}=4.7 \mathrm{kG}, \lambda_{W}=$ $0.88 \mathrm{~cm}, L_{W}=60 \mathrm{~cm}$, and $\rho_{0}=1.6 \times 10^{13}$ electrons per $\mathrm{cm}^{3}$ (which corresponds to a peak current of $100 \mathrm{~A}$, and e-beam diameter of $0.02 \mathrm{~cm}$ ), we get $G_{\max } \sim 0.9$.

The small signal gain expression above is an approximate one which assumes a monoenergetic electron beam and it is valid when $G_{\max }$ itself is small. A simple 1-D simulation code [15] with energy spread, angular spread and saturation effects shows that the FEL will reach saturation in approximately 50 round trips. The round trip time in our resonator about 25 ns, so 50 round trips is well inside the $2.5 \mu$ s electron macropulse.

We have also computed the small signal gain with the 3-D numerical model of a freeelectron laser, FELOPT [16]. We assume an e-beam that is circularly symmetric and whose charge distribution falls off parabolically with increasing radius, truncated at $0.02 \mathrm{~cm}$. The peak small signal gain (SSG) computed with the $3-\mathrm{D}$ model is $46.5 \%$ per pass. The SSG drops below $20 \%$ per pass when the normalized (transverse) beam emittance, $\epsilon_{N}$ is $20 \mathrm{~mm}$-mrad (for $\gamma=100$ ). Thus, if the e-beam is properly matched into the undulator, it is expected that there should not be any significant gain degradation in the FEL.

The models show that the peak gain drops by approximately $20 \%$ when the energy spread $\delta \gamma / \gamma=0.5 \%$ (FWHM). The energy spread is expected to be well within this limit. 


\section{THE SUPERFERRIC UNDULATOR}

One of the goals of this research effort is to develop compact FEL systems capable of producing short wavelength radiation, tunable over a broad band. A key to achieving this goal is the mini-undulator technology being developed at Brookhaven using tunable, superconducting magnets [5]. The key parameters of the undulator being built for this experiment are a period $\lambda_{W}=0.88 \mathrm{~cm}$, a length $L_{W}=61.6 \mathrm{~cm}$ (70 periods) and a full gap spacing of $0.44 \mathrm{~cm}$.

The full length undulator consists of three $20 \mathrm{~cm}$ sections plus half period end correction units. Each section is machined from solid steel stock by computer controlled WEDM (Wire Electric Discharge Machining). This technique results in mechanical tolerances better than $10 \mu \mathrm{m}$ for the pole-to-pole gap variations and the reproducibility in the periodicity necessary for the random field error to be less than $0.3 \%$ as machined. The elimination of the field adjustment process reduces significantly the cost of the undulator.

The wire is wound in $2.30 \mathrm{~mm}$ wide channels with 18 turns in four layers. The number of turns alternates between five and four turns within a continuous winding along the whole undulator. The wire used is a 54-filament niobium-titanium superconductor with a copperto-superconductor ratio of 1.3. The outer diameter of the wire is $0.440 \mathrm{~mm}$ including a thin formvar insulation. The critical current as specified by the the manufacturer is $142 \mathrm{~A}$ at $5 \mathrm{~T}$ and $4.2 \mathrm{~K}$, (which corresponds to a critical current density of $2800 \mathrm{~A} / \mathrm{mm}^{2}$ ). A much higher current is obtained at the relatively low ainbient magnetic field characteristic of this design.

The sections are wound independently and assembled next to each other within a box frame. A stainless steel precision spacer between the two yokes defines the gap. Then the assembly is inserted into the cryostat.

In cryogenic tests of one $20 \mathrm{~cm}$ section, the measured peak field on axis was $0.52 \mathrm{~T}$. The limit is given by the critical current $I_{c}$ of the magnet $(246 \mathrm{~A}$ ), which is near the measured short sample current at the operating field. The measured peak field rms error was $0.3 \%$ over the 10 central periods. The second field integral over this section shows a $5 \mu \mathrm{m}$ walk.

The FEL cryostat was designed and built by the Janis Research Co. It is configured 
as a vertically oriented cylinder having both a diameter and height of approximately one meter. The beamline horizontally pierces the cylindrical portion of the cryostat at two 0.33-m diameter flanged ports.

Internally, the superconducting undulator is immersed in liquid helium at atmospheric pressure. A liquid nitrogen cooled heat shield is also provided. The volume of the cryogens will maintain cooling for 48 hours between refills. Liquid helium and nitrogen levels, as well as the insulating vacuum are monitored by integral instrumentation. Electric power is supplied through a feedthrough centrally mounted on the top flange

Access to the undulator is obtained through the beamport flanges and by removing the bottom flange. To facilitate this, the support stand has detachable jacks and casters which allow it to be moved off its mounts out of the beamline.

\section{THE OPTICAL RESONATOR}

The resonator consists of a standing wave, stable optical cavity with one totally reflecting dielectric-coated mirror and one partially transmitting mirror for laser output coupling. Both optical elements are provided with remote two-axis, tip/tilt alignment control. In addition, the high reflector is equipped with a linear positioner to permit remote cavity-length control. The optical cavity design and some of the alignment and control systems are very similar to those used earlier in Rocketdyne's FEL experiments at Stanford [17] [18]. Alignment requirements for the BNL resonator are, however, more stringent due to the shorter optical wavelength.

The optical resonator [19] must provide adequate feedback and mode control to sustain laser oscillations and maintain output stability. It must also maintain maximal spatial and temporal overlap between the e-beam and the mode and be able to withstand the high optical power in the cavity.

The mirror radius of curvature is chosen to match the Rayleigh length of the laser mode, to the length of the undulator. Using Gaussian beam analysis, the optimum waist radius $w_{0}$ 
is readily calculated $[20] w_{0}=\left[\lambda L_{W} /(2 \pi)\right]^{1 / 2}$. For $\lambda=0.5 \mu \mathrm{m}$, we get $w_{0}=0.022 \mathrm{~cm}$. For a symmetric resonator of length $\mathrm{L}=367.65 \mathrm{~cm}$, the radius of curvature of the mirrors is then $188.72 \mathrm{~cm}$, corresponding to a Rayleigh length $Z_{R}=30.0 \mathrm{~cm}$.

An outcoupling of $1 \%$ to $5 \%$ will be used in initial experiments. Once efficient lasing is obtained, a larger output coupling will be used. The peak intracavity irradiance on the mirrors may be as high as $8 \mathrm{GW} / \mathrm{cm}^{2}$ with a macropulse energy up to $50 \mathrm{~mJ}$ for $5 \%$ outcoupling. Results of steady-state simulations show adequate small-signal gain (46\% per pass) for $10 \%$ output coupling fraction. A broadband ( $\pm 8 \%$ for outcoupling between 5 and $10 \%$ ) dielectric mirror will be used, with a center wavelength of $535 \mathrm{~nm}$ to permit the use of a green He-Ne laser (543 nm) for alignment and a pulsed, doubled YAG laser (532 nm) for initial adjustment of resonator length.

The resonator stability parameter is $\mathrm{g}=1 \cdot \mathrm{d} / \mathrm{R}=-0.95$. Since near concentric ( $\mathrm{g}$ near -1) optical cavities are particularly sensitive to alignment errors, iterative resonator computations were done as a function of the mirror misalignment. A $20 \mu \mathrm{rad}$ mirror tilt was found to be acceptable. The steady state cavity power is $243 \mathrm{MW}$ for a perfectly aligned cavity and $210 \mathrm{MW}$ for a $20 \mu \mathrm{rad}$ tilt. The 3-D intensity distribution at the center of the undulator and the output mirror show that for a $50 \mu \mathrm{rad}$ tilt the mode is displaced and slightly distorted, but otherwise well formed. A Rayleigh length of $18.53 \mathrm{~cm}$ was also evaluated. The alignment tolerance with this value was considered unacceptable.

Computations were also performed to evaluate the sensitivity of the SSG and power to transverse displacements of the e-beam relative to the undulator axis (with the undulator and resonator axes coaligned). Results indicate that a displacement of $10 \%$ of the rms ebeam radius causes the power to drop by $20-25 \%$. Thus, the e-beam (and the resonator optical axis) must be centered to the undulator axis within $\pm 20 \mu \mathrm{m}$. 


\section{PRECISION ALIGNMENT SYSTEM}

The stringent alignment requirements discussed above will be satisfied with precision mirror mounts having independent, remotely controlled, rotation about two axes with an estimated resolv 'ion of $\pm 2 \mu \mathrm{rad}$.

In the initial alignment and during FEL operation, the undulator axis, the electron beam line and the resonator axis should form a single line. This alignment must be performed through a 4-mm-diameter bore inside a cryostat. Due to these restrictions and the compact size of the laser, these alignment functions are accomplished through a single port, using compact, multi-position, multi-function viewports [21], one near each end of the undulator.

The multi-function viewport has three positions. In position (i), the device permits resonator alignment by inserting a 50/50 beam splitter (at 45 degrees to the undulator axis) into the laser cavity, permitting visual access into an otherwise inaccessible chamber. In this position, an alignment beam from a mode-matched, green HeNe laser (nominally at right angles to the resonator axis) can be injected into the resonator. The primary and secondary reflections from the two resonator mirrors, along with fiducial marks at the ends of the undulator, are then used to co-align the resonator and the undulator axes. Normal laser operation occurs in position (ii), which permits free passage of the laser and e-beams. In position (iii) a metallic, phosphorescent screen images the e-beam position and shape on a video camera through the bottom of the view port. The alignment laser beam may be injected through the outcoupler for viewing on this screen. The e-beam and alignment laser beam can be coaligned by viewing them alternately or simultaneously. Then the trajectory of the e-beam can be aligned to the undulator axis by iterating between the two viewports at each end of the undulator. As the device is cycled among the three positions, the beam splitter orientation is critical in the first position, and screen placement is critical in the third position, while in the intermediate position neither is critical. Repeatability is assured ( $\leq 1 \mu \mathrm{rad}$ in orientation, and \pm 0.0005 inch in location) by utilizing kinematic design principles. 


\section{OPTICAL DIAGNOSTICS}

The FEL output beam will be directed to optical tables outside the radiation shielding for measurements of the beam's time dependence, pulse energy, spectrum and mode. A pop-in mirror will extract spontaneous emission. The FEL's radiation growth will be followed by a photomultiplier up to $10 \mathrm{~nJ}$ and then by pyroelectric joulemeters.

A CCD video camera and digital frame grabber will measure the two-dimensional spatial profile of the beam. The camera is sufficiently sensitive to measure the profile of the spontaneous beam, integrated over a linac macropulse.

It is desirable to follow the temporal evolution of the FEL output spectrum with a micropulse resolution in a single macropulse. We have been investigating the use of a highspeed rotating polygonal mirror to deflect different micropulses to different heights on a spectrometer entrance slit. A spectrometer designed to minimize optical aberration can image the spectra of these micropulses on different lines of the video camera. The dynamicrange requirement is reduced by the use of a spatially varying attenuator at the entrance slit.

The same rotating mirror can be used to study the evolution of the transverse mode structure. A one-dimensional slice of the beam can be deflected across the video camera to show the change in the mode due to optical guiding or to walking of the beam because of resonator misalignment.

The optical time dependent measurements described above can be correlated with timedependent electron-beam energy spectra mentioned above, revealing the micropulse to mi. cropulse evolution. Since both optical and electron energy spectra can be acquired in a single macropulse, the macropulse to macropulse stability does not affect this data.

Finally, we also intend, in an experiment with Columbia University, to study the growth of spikes and sidebands in the saturated FEL. We are considering the construction of a single-shot autocorrelator with a resolution of $100 \mathrm{fs}$ [22]. The temporal structure of one micropulse, selected by a Pockels cell, can be measured and compared to the evolution of sidebands in wavelength. 


\section{PROJECT STATUS}

The ATF is in an advanced state of construction with the beam transport elements up to the experimental hall in place. The injection system is now operational, and provides 10 ps (FWHM) pulses of $2 \mathrm{nC}$. The operation of the facility is carried out from the control room by means of a computerized process control.

All undulator sections have been machined within the specified tolerance of $10 \mu \mathrm{m}$ for the critical parameters of gap and periodicity. Superconducting tests of a $20 \mathrm{~cm}$ section are under way. The undulator cryostat has been built and the full $60 \mathrm{~cm}$ undulator should be finished and tested in autumn 1991.

The major tasks remaining are: commissioning of the $50 \mathrm{MeV}$ linac, completion of the 100 pulse train photocathode laser, final installation of the beam transport hardware in the experimental hall and commissioning. The ATF commissioning is scheduled to be completed and experiments to begin in spring 1992.

\section{References}

[1] I. Ben-Zvi, L.F. Di Mauro, S. Krinsky, M.G. White, L.H. Yu, K. Batchelor, A. Friedman, A.S. Fisher, H. Halama, G. Ingold, E.D. Johnson, S. Kramer, J.T. Rogers, L. Solomon, J. Wachtel and X. Zhang. "Proposed UV-FEL User Facility at BNL" Proceedings of the 1991 International FEL Conference, Santa Fe, NM.

[2] I. Ben-Zvi, K.M. Yang and L.H. Yu, "The 'Fresh-Bunch' Technique in FELs" Proceedings of the 1991 International FEL Conference, Santa Fe, NM.

[3] I. Ben-Zvi, A. Friedman, C.M. Hung, G. Ingold, S. Krinsky, L.H. Yu, I.S. Lehrman and D. Weissenburger, "Design of a Harmonic Generation Experiment at BNL" Proceedings of the 1991 International FEL Conference, Santa Fe, NM.

[4] K. Batchelor, I. Ben-Zvi, R. Fernow, J. Gallardo, H. Kirk, C. Pellegrini, A. van Steenbergen and A. Bhowmik, "A microundulator free-electron laser at the Brookhaven 
Accelerator Test Facility”, Nucl. Instr. and Meth., A296 (1990) 239-243.

[5] I. Ben-Zvi, R. Fernow, J. Gallardo, G. Ingold, W. Sampson and M. Woodle, "Performance of a Superferric, High Field Subcentimeter Undulator" Proceedings of the 1991 International FEL Conference, Santa Fe, NM.

[6] M. E. Couprie, M. Billardon, M. Velgne, C. Bazin, J. M. Ortega, R. Prazeres and Y. Petroff, "Free-electron laser oscillation on the Super-ACO Storage ring at Orsay", Nucl. Instr. and Meth., A296 (1990) 13-19.

[7] G. N. Kulipanov, V. N. Litvinenko, I. V. Pinaev, V. M. Popik, A. N. Skrinsky, A. S. Sokolov and N. A. Vinokurov, "The VEPP-3 Storage-Ring Optical Klystron: Lasing in the visible and the ultraviolet regions", Nucl. Instr. and Meth., A296 (1990) 1-3.

[8] D. R. Shoffstall, "Visible wavelength free-electron laser", Proc. Int. Conf. on Lasers, Lake Tahoe, NV, 1988 (STS Press, McLean, VA, 1989), p. 171.

[9] I. Ben-Zvi, "The BNL Accelerator Test Facility and Experimental Program", Proceedings of the 1991 Particle Accelerator Conference, San Francisco CA.

[10] K. Batchelor, I. Ben-Zvi, R.C. Fernow, J. Fischer, A.S. Fisher, J. Gallardo, X. Jialin, H.G. Kirk, R.G. Malone, Z. Parsa, R.B. Palmer, T. Srinivasan-Rao, J.T. Rogers, J.F. Sheehan, T.Y.F. Tsang, S. Ulc, A. van Steenbergen, M. Woodle, R.S. Zhang, "Development of a high brightness electron gun for the Accelerator Test Facility at Brookhaven National Laboratory", presented at the European Particle Accelerator Conference, Rome, Italy, 1988.

[11] K. Batchelor, I. Ben-Zvi, R. Fernow, J. Fischer, A. Fisher, J. Gallardo, G. Ingold, H. Kirk, K.P. Leung, R. Malone, K. McDonald, I. Pogorelsky, D. Russell, T. SrinivasanRao, J. Rogers, T. Tsang, J. Sheehan, S. Ulc, X.J. Wang, M. Woodle, J. Xie and R. Zhang, "Performarie of the Brookhaven Photocathode RF Gun", Proceedings of the 1991 International FEL Conference, Santa Fe, NM. 
[12] I. Ben-Zvi, J. Xie and R. Zhang, "Feed Forward RF Control System of the Accelerator Test Facility", Proceedings of the 1991 Particle 4ccelerator Conference, San Francisco CA.

[13] R. Walker, "Electron Beam Focussing Effects and Matching Conditions in Plane Periodic Magnets", Nucl. Instr. and Meth. A214, 497, 1983.

[14] W. B. Colson, "The nonlinear wave equation for higher harmonics in free-electron lasers”, IEEE J. Quant. El., QE-17, 1981, p. 1417.

[15] J. Gallardo and C. Pellegrini, "Optical Klystron Configuration for a High Gain X-ray Free-Electron Laser", Optics Comm. 77, 45 (1990)

[16] A. Bhowmik, R. A. Cover and R. H. Labbe, "Three dimensional modeling of free-electron lasers using rigorous wave propagation", Proc. SPIE, 642, 10 (1986).

[17] A. Bhowmik, M. S. Curtin, W. A. McMullin, S. V. Benson and J. M. J. Madey, B. A. Richman and L. Vintro, "First operation of the Rocketdyne/Stanford free-electron laser", Nucl. Instr. and Meth., A272 (1988) 10-14.

[18] A. Bhowmik, M. S. Curtin, W. A. McMullin, S. V. Benson and J. M. J. Madey, B. A. Richman and L. Vintro, "Initial results from the master oscillator/power amplifier experiment", A296 (1990) 20-24.

[19] A. Bhowmik, N. Lordi, I. Ben-Zvi and J. Gallardo, "Resonator Design for a Visible Wavelength Free-Electron Laser", Proceedings of the 1990 International Conference on Lasers, San Diego CA.

[20] R. A. Cover and A. Bhowmik, "Astigmatic effects in linearly polarized, tapered-wiggler, free-electron lasers", Proc. Int. Conf. on Lasers, San Francisco, CA (1983) (STS Press, MiLean, VA, 1984) p.263.

[21] A. Bhowmik and J. Brown, "A compact, multi-function position monitor for precision alignment of laser/particle beams", Rocketdyne patent disclosure \#91R070, Feb. 1991. 
[22] F. Salin, P. Georges, R. Roger and A. Brun, "Single shot measurement of a 52-fs pulse" Appl. Optics 26, 4528 (1987). 


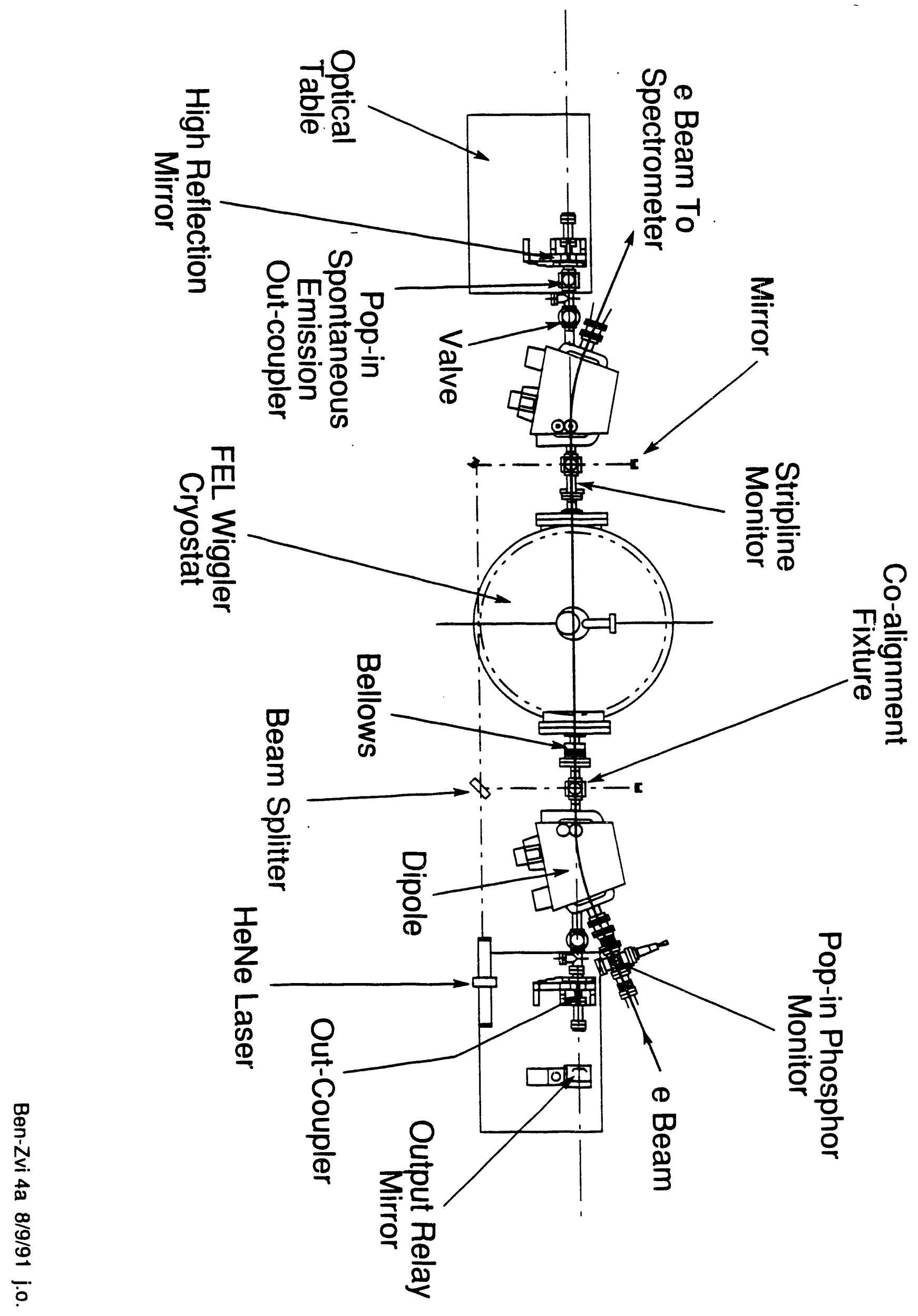



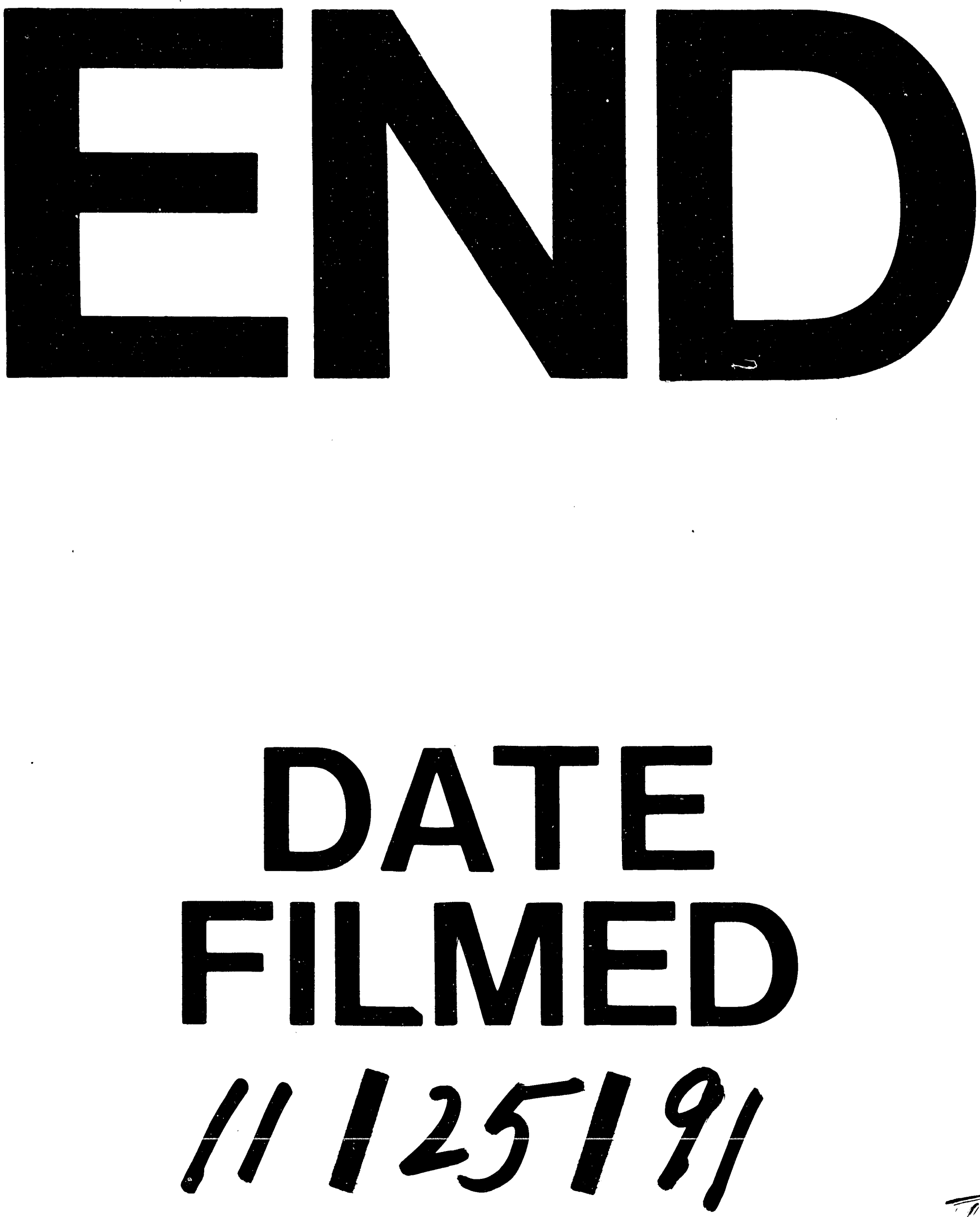
